Research Article

\title{
Constructing Sports Multi-Index Data Analysis Based on 5G IoT Technology
}

\author{
Hui Wang $\mathbb{D}^{1,2}$ and Ben Zhao' \\ ${ }^{1}$ College of Physical Education and Health, East China Normal University, Shanghai 200241, Shanghai, China \\ ${ }^{2}$ College of Physical Education, Xinjiang Normal University, Urumqi 830054, Xinjiang, China
}

Correspondence should be addressed to Hui Wang; 52201000018@stu.ecnu.edu.cn

Received 13 June 2021; Accepted 6 August 2021; Published 18 August 2021

Academic Editor: Sang-Bing Tsai

Copyright (c) 2021 Hui Wang and Ben Zhao. This is an open access article distributed under the Creative Commons Attribution License, which permits unrestricted use, distribution, and reproduction in any medium, provided the original work is properly cited.

\begin{abstract}
The arrival of the new era and the development of 5G Internet of Things (IoT) technology have made our lives and work easier and more convenient. The vigorous development of the IoT has been applied in many fields, among which, especially the data mining technology of the IoT ushered in the spring of this era of information explosion. Full application of data mining technology can provide real data well. Application analysis provides value and decision support. In order to apply 5G Internet of Things technology to the sports industry to help study the multi-index data of various sports activities so as to better help modern people have a healthy sports concept, Nemo builds relevant data analysis based on 5G Internet of Things technology. This article analyzes the research on the construction of sports multi-index data based on 5G IoT, makes full use of the IoT to mine sports-related data, and launches a multi-index discussion on it. First, the literature data method is adopted to learn the theoretical knowledge of IoT, artificial neural network, deep learning, etc., and establish a sports multi-index data analysis research model based on machine learning and massive data processing technology. Finally, for modern people, sports hobbies, types, exercise duration, exercise heart rate, and other aspects are analyzed. The results show that modern people prefer aerobic exercise, especially jogging and cycling, accounting for $47 \%$ and $41 \%$, and the proportion of people who spend more than 60 minutes in the gym is as high as $48 \%$. This shows that even though most people are busy at work, they still realize the importance of physical exercise and are willing to do sports.
\end{abstract}

\section{Introduction}

Sport is an indispensable part of people's lives. If you want to get a good body and a healthy body, you must do sports. Sports are not only exclusive to athletes but also a way for most people to exercise. With the development of society and the improvement of material level, most people's awareness of fitness has also been improved, and more and more attention is paid to sports, not only in their attention to sports competitions but also in sports in daily life. Physiologically, physical exercise is conducive to the growth of human bones and muscles, enhances cardiopulmonary function, improves the functional status of the blood circulatory system, respiratory system, and digestive system, is conducive to the growth and development of the human body, improves disease resistance, and enhances the adaptability of organisms.

The Internet of Things technology has developed vigorously, first of all, its data processing technology, that is, data mining and analysis technology, which can be applied to all walks of life, from facial recognition technology to aircraft carrier manufacturing. It can output, measure, capture, and send data through some information collection equipment (ultrasonic sensors, radio frequency identification, laser scanners, and other global positioning systems, etc.). These data have a wide range of systems and collections. As long as it is a detected object, data can be collected. Then, it can be sent to the server for monitoring. The Internet of Things uses various intelligent computer technologies, such as information processing technology, cloud 
computing technology, data mining technology, and analysis tools, and can perform intelligent data analysis, calculation, and statistics on collected data.

Many scholars at home and abroad have conducted relevant analyses on the construction of multiple indicators of sports based on 5G Internet of Things technology. Balaji proposes that the interaction between customers and Internet of Things retail technology will lead to the cocreation of value. These responses come from 289 IoT users in the retail environment. The results of PLS equation modeling show that usability, superior function, aesthetics, and existence are the key factors to create the technical value of IoT retail. But it still has some limitations [1]. Park's study aims to explore the influence of social support on winter sports athletes' self-determination and athletic performance. Using a purposeful sampling method, 217 athletes from the Korean Olympic Committee Winter Olympics were selected as the research objects. The data are collected on a self-rating scale and are based on the self-management questionnaire used in previous studies. Methods such as frequency analysis, factor analysis, $t$-test, one-way analysis of variance, correlation analysis, and multiple regression analysis are used to solve the problems in the research. First, there are significant differences in social support, self-determination, and athletic performance in gender, national athlete experience, athlete career, and athlete ability. Second, social support has a significant impact on self-determination. Third, social support has a significant impact on sports performance. Fourth, self-determination has a significant impact on athletic performance. Therefore, various ways to increase social support and the right to self-determination must be effectively developed. They will help improve athletic performance and athlete satisfaction [2]. Palatella performance analyzes the potential of $5 \mathrm{G}$ technology in the Internet of Things from two aspects of technology and standardization and proposes that $5 \mathrm{G}$ cellular system realizes the connection technology with cost-effectiveness, high reliability, and scalability, but there are great differences in the application and demand of various communication technologies [3].

This article analyzes the sports multi-index data based on $5 \mathrm{G}$ Internet of Things technology. In the method part, this article introduces the technical methods and data mining technology of 5G Internet of Things. In the analysis part, a comparative analysis of sports index data based on the Internet of Things and sports multi-index data based on 5G IoT is carried out. The innovations of this paper are as follows: (1) combine qualitative research with quantitative research and fully analyze the research data; (2) combine theoretical research with empirical research, and combine sports on the basis of IoT technology model, the actual situation in the empirical investigation.

\section{Method of Constructing Sports Multi-Index Data Based on 5G IoT}

2.1.5G IoT Technical Methods. The IoT refers to the existing terminal equipment and facilities sensors, mobile terminals, industrial systems, numerical control systems, intelligent home devices, video surveillance systems, and "inherent intelligence system," the comprehensive integration $[4,5]$. It can be connected to each other through various wireless or wired long-distance or short-distance communication networks (M2M), cloud-based applications and SaaS function integration, and the internal network and external network, and Internet environment can be safely controlled, and even carry out personalized online monitoring, realize traceability, alarm connection, data remote management, positioning, real-time management, scheduling, standardization, and other functions, and implement integrated management of "management, control, and operation" on data [6].

IoT applications have the following basic technologies:

(1) Sensor technology: it is also the basic technology of computer applications. It is a sensor that converts analog signals into digital signals [7].

(2) RFID tag: it is also a kind of sensor technology. It is an integrated technology that combines radio frequency technology and embedded technology [8].

(3) Embedded system technology: it is slowly changing people's lives and promoting the development of industry and agriculture in our country [9].

(4) Intelligent technology: it is one of the key technologies of the IoT, and the most common application is artificial intelligence. It is to implant or embed the intelligent system into the target object through other means so that the target object has a certain degree of intelligence and can realize effective dialogue and information exchange like people [10].

The typical architecture of the Internet of Things is divided into three levels from bottom to top, perception layer, network layer, and application layer [11, 12]. The perception layer is the basic ability to perceive the comprehensive perception of the Internet of Things. The application layer provides various applications, combining Internet technology and the needs of IT departments to achieve a wide range of intelligent application solutions. What is important is the integration of the industry, the development and utilization of information resources, lowcost solutions, and high-quality and secure information. Ensure and develop effective business models [13, 14].

Figure 1 shows the key technology roadmap of the IoT. This key technology roadmap includes key technologies such as radio frequency identification technology, QR codes, sensors, IPv6, cloud services, cloud storage, and cloud computing.

2.2. Data Mining Technology. Data mining refers to the process of finding hidden information in large amounts of data through algorithms. Data mining is usually related to computer science, and its purpose is to achieve it through various methods such as information retrieval, machine learning, expert systems (based on previous experience rules), standard recognition, and other network statistics, analysis, and processing $[15,16]$. Whether in finance, medical care, or various fields of society such as telecommunications and education, massive amounts of data are 


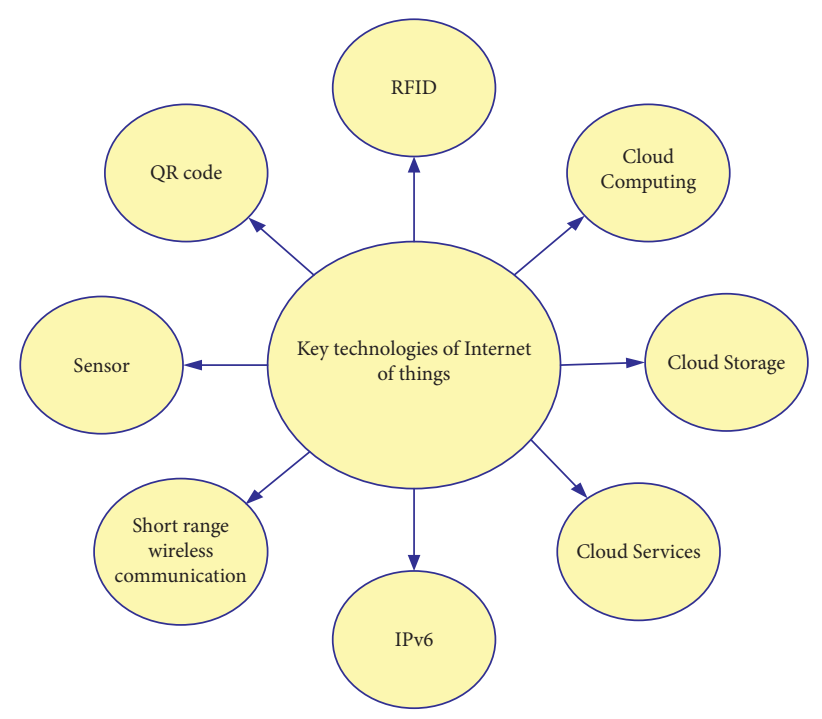

Figure 1: Key technology roadmap of IoT.

generated at every moment. Due to too many uncertain factors in society, the types of data processed are becoming more and more complicated, even if it is used. Computer assistance is still limited in traditional processing methods and solving practical problems. However, solving big data problems through data mining technology opens up another way.

The data type can be structured, semistructured, or heterogeneous $[17,18]$. The method of discovering knowledge may be mathematical, nonmathematical, or inductive. The knowledge finally discovered can be used to manage information, optimize problems, support decision-making, and maintain the data itself. The data types that can be mined include both relational databases and structured data, as well as data warehouses, text, multimedia data, spatial data, time axis data, and organizational data [19, 20].

Considering the data itself, data mining typically requires eight steps, such as cleaning the data, data conversion, data extraction process, evaluation criteria, and other knowledge representation [21, 22].

(1) Information collection: based on the data analysis of the moving object, the summary of the information characteristics is required to select the appropriate information collection method.

(2) Data integration: collect logical data or physical data of various sources, forms, and characteristics, and provide comprehensive general data usage methods.

(3) Data protocol: the standardized processing of data, which can greatly save data processing time and maintain the integrity of the original data.

(4) Data cleaning: the information in the database has many characteristics. You only need to select the required data and rearrange it and then save it in the database.

(5) Data conversion: through smooth data collection, generalization, and standardization, it is converted into a form suitable for data extraction. Some important data and data classification in the data hierarchy are also transformed through data.

(6) Data mining: choose appropriate analysis tools, use statistical methods, and get a visual analysis report.

(7) Evaluation model: from a business point of view, industry experts will verify the correctness of results.

(8) Knowledge expression: the analytical information extracted from the data is visualized.

In data mining, at least $60 \%$ of the cost is used for the information collection stage, and at least $60 \%$ of the energy and time is used for data preprocessing [23].

\section{Based on 5G IoT to Construct the Analysis and Research Experiment of Sports Multi- Index Data}

3.1. Research Model of Sports Multi-Index Data Analysis Based on Machine Learning. The data analysis model is mainly carried out from the three steps of data collection, data accumulation, and data preprocessing. This article first proposes a sports multi-index data analysis research model based on machine learning, which makes good use of machine learning technology in 5G IoT [24].

Machine learning is a subject, including probability theory, statistics, methodology, critical analysis, and other algorithmic complexity theory and other issues. In order to acquire new knowledge and skills, reorganize the existing knowledge structure, and continuously improve its own performance, it specializes in computer simulation or methods of improving human learning behavior. This is the core of artificial intelligence and the basic method to realize computer intelligence [25].

The research directions of traditional machine learning mainly include research on decision trees, random forests, artificial neural networks, and Bayesian learning [22]. Here is an introduction to two machine learning algorithms, artificial neural networks and deep learning. 
3.1.1. Algorithm of Artificial Neural Network. A neural network is a computer system in which nodes are connected to each other. Its nodes function like neurons in the human brain. These neurons process information and send each other. Each neural network is a series of algorithms that identify possible relationships in datasets by simulating human functional processes [26].

Its calculation process is as follows:

First, start from the input layer of the neural network model to the hidden layer. The total input of the $j$ th node of the hidden layer from the input layer is as follows:

$$
h_{1}=\sum_{i=1}^{i=14} W_{i j} \times x_{i}
$$

Among them, the range of $i$ is the node number $1-14$ of the input stage, and the $i$ th node of the input layer and the $j$ th node of the hidden layer are the weights between nodes. Numerical value: the output port of the $j$ th node to the hidden layer is as follows:

$$
V_{j}=f\left(h_{j}\right) \text {. }
$$

The sigmoid function is as follows:

$$
f(x)=\frac{1}{1+e^{-x}} \text {. }
$$

Among them, the range of $j$ is $1-4$, which is the number of hidden layer nodes.

The output layer comes from the input of the hidden layer.

$$
p_{k}=\sum_{j=1}^{j=4} w_{j k} \times v_{j}
$$

Among them, $j$ represents the number of hidden layer nodes and $k$ represents the number of output layers. Substitute the above formula to get

$$
p_{k}=\sum_{j=1}^{j=4} w_{j k} \times f\left(h_{j}\right)=\sum_{j=1}^{j=4}\left[w_{j k} \times f\left(\sum_{i=1}^{i=14} w_{i j} \times x_{i}\right)\right] .
$$

Then the output of the output layer is as follows:

$$
Y_{k}=f\left(P_{k}\right)=f\left\{\sum_{j=1}^{j=4} W_{j k} \times f\left(\sum_{i=1}^{i=14} W_{i j} \times x_{i}\right)\right\} \text {. }
$$

3.1.2. Deep Learning Algorithm. Learn the depth and connotation of data [27]. The information obtained in the learning process is very useful for interpreting data [28]. Deep learning is a complex machine learning algorithm. The effect on speech and image recognition is far more than previous technologies. Deep learning in retrieval, data mining, machine learning, machine translation, natural language processing, learning and multimedia, voice, proposals, personalization, and other related fields has made many achievements. This is the reason why technologies related to artificial intelligence have made great progress.

The basic algorithm of deep learning is RBM algorithm.

$\mathrm{RBM}$ is a special Markov random field structure composed of a visible $v$ layer and a hidden $h$ layer. RBM is based on the energy probability model in statistical engineering. If the model parameters are specified, RBM is the general distribution of the energy dependence of the $v$-layer unit and the $h$-layer unit.

$$
P(x)=\frac{e^{-E(v, h \mid \delta)}}{Z} .
$$

Expression (7) only evaluates the RBM state of the twolayer structure (visible layer $v_{m}$ and hidden layer $h_{n}$ ). $Z$ is the normalized convergence coefficient, expressed by the mathematical double accumulation method: $Z=\sum(v) \sum(h)$ $e-\sum(x)$, for two RBM level models, it can be described by the Bernoulli-Bernoulli energy equation. The energy function based on the RBM training model is expressed as follows:

$$
E(v, h \mid \delta)=-\sum_{m=1}^{j} b_{m} v_{m}-\sum_{n=1}^{i} c_{n} h_{n}-\sum_{n=1}^{i} \sum_{m=1}^{i} w_{m n} v_{m} h_{n}
$$

where $i$ and $j$ are the unit datasets of the visible layer $v_{m}$ and the nondisplay layer $h_{n}$, respectively. The weight $h$ is the connecting axis. When $v_{m}$ follows a specific probability distribution, the visual unit $v_{m}$ will be given according to the RBM training model and the independent activity state parameters of each $h_{n}$ unit. Among them, the conditional probability formula of $v_{m}$ and $h_{m}$ is as follows:

$$
\begin{aligned}
& P\left(h_{n}=1 \mid v ; \delta\right)=\theta\left(\sum_{n=1}^{n} w_{m n} v_{m}+b_{m}\right), \\
& P\left(v_{m}=1 \mid h ; \delta\right)=\theta\left(\sum_{n=1}^{n} w_{m n} h_{n}+c_{n}\right) .
\end{aligned}
$$

$x$ in the above two formulas is the threshold function Sigmoid, and the expression is as follows:

$$
\theta(x)=\frac{1}{1+e^{-x}}
$$

It is only applicable to the RMB 2-layer structure model. When the model structure exceeds 2 layers, the energy equation becomes invalid. The training model of the multilayer structure is realized by the Gauss-Bernoulli equation. The energy equation is as follows:

$$
E(v, h \mid \delta)=-\frac{1}{2} \sum_{m=1}^{j}\left(v_{m}-b_{m}\right)^{2}-\sum_{n=1}^{i} c_{n} h_{n}-\sum_{n=1}^{i} \sum_{m=1}^{j} w_{m n} v_{m} h_{n} .
$$

The conditional probability distribution of continuous actual value RMB is as follows: 


$$
\begin{gathered}
P\left(h_{n}=1 \mid v ; \delta\right)=\theta\left(\sum_{n=1}^{n} w_{m n} v_{m}+b_{m}\right), \\
P\left(v_{m} \mid h ; \delta\right)=N\left(\sum_{n=1}^{n} w_{m n} h_{n}+c_{n}, 1\right) .
\end{gathered}
$$

In the formula, $v_{m}$ is the continuous actual value, 1 is the variance, and $w_{m n} h_{n}$ is the mean value.

The nature of RBM is that when the state of the neurons in the visible layer is given, the activation conditions of the neurons in the hidden layer are independent; on the contrary, when the state of the neurons in the hidden layer is given, the activation of the neurons in the visible layer is also conditionally independent.

\subsection{Sports Multi-Index Data Analysis Research Model Based} on 5G IoT Massive Data Processing Technology. The basic analysis of the large-scale information processing technology of the Internet of Things includes the analysis of various network test equipment. These data are processed efficiently and quickly, and valuable information is obtained through the classification and comprehensive analysis of important information, thereby helping users to make wise decisions. Based on this, this paper designs a sports multi-index data analysis research model based on 5G Internet of Things massive data processing technology. This model uses multilevel data comprehensive analysis to establish different data sources and multilevel comprehensive analysis requires data to be measured. To solve various problems, it is necessary to merge and effectively use data sources.

\subsubsection{The IoT Stores, Retrieves, and Queries Sports Data.} The IoT widely develops and uses detection equipment to collect and generate large amounts of real-time data and multi-source data in multiple details and multiple latitudes, and its data range may reach $\mathrm{TB}$ or $\mathrm{PB}$ level. A large amount of data collection and questioning are very important for centralizing and effectively processing these large amounts of data in Internet surveys for storage and other technological development. In order to achieve the perfect combination of these technologies and analysis technologies, the necessary data effective management and comprehensive adjustment will be executed in real time. Storage mechanism: the principle of closed storage is adopted to provide general short view nodes. Each data archiving node notifies the global summary view node of data dispersion. The request for the problem is first sent to the global shortcut viewer node, and it can be quickly found in the data. To avoid many problems with the web button, design a data recovery mechanism when storage disks lose capacity, dynamically adjust the data life cycle of various data types, and perform query functions based on historical statistics and current system storage capacity. Data beyond the life cycle will be replaced by new data. Therefore, it is recommended to use irrelevant database technology to store the data in a single network node. Indexing mechanism research: IoT data includes time flow data and spatial flow data.
According to the type of archived data, search-optimized indexes are automatically created. For time flow data, the main problem type is spatial problems. For spatial flow data, problem functions can be used to find all suitable items in a specific area, and multidimensional indexing technology can be used to determine the best indicators, so the system can meet efficiency and processing requirements in real time.

3.2.2. Technical Analysis of the Real-Time Database of the IoT. Distributed storage technology database is a combination of previous database technology and network technology. A distributed database is a dataset distributed in the physical space of each node in a computer network, but it can logically belong to the same system. This includes local site database management system, global management system, database, global data query, and communication management. It is responsible for the creation and management of the local database. Realize website autonomy, execute local applications and other functions, provide distributed transparency, adjust the execution of global events, adjust various local database management systems, and ensure database global consistency, information synchronization, and other functions.

Figure 2 is a schematic diagram of the database collection, query, and analysis system. This system first collects data and then uses a series of data management tools to collect understandable data, existing data, and historical data. Finally, use analysis tools, reporting tools, etc. for these data.

\section{Constructing Sports Multi-Index Data Based on 5G IoT}

4.1. Sports Index Data Based on IoT Database. The most common classification in sports is divided into aerobic exercise and anaerobic exercise. Aerobic exercise refers to physical exercise performed by the human body under the condition of sufficient oxygen supply. That is, during exercise, the body's intake of oxygen is equal to its demand, reaching a physiological equilibrium state. When the body needs a lot of energy in an instant, and under normal circumstances, aerobic metabolism cannot meet the body's needs at this time, so sugar undergoes anaerobic metabolism to quickly produce a lot of energy. Exercise in this state is anaerobic exercise. Among them, aerobic exercise includes yoga, swimming, cycling, jogging, Tai Chi, square dance, etc.; anaerobic exercise includes sprinting, push-ups, long jump, high jump, tug of war, diving, etc. This article draws the following sports index data based on 5G IoT technology.

As can be seen from the data in Table 1, the proportion of the number of people doing aerobic exercise is more than the number of people doing anaerobic exercise. Among them, the number of people jogging and cycling is relatively high, $47 \%$ and $41 \%$, respectively. Among the daily exercise methods, jogging is the most effective and does not require other sports equipment. It can be convenient to exercise when going out, so the number of people doing this exercise is the largest. 


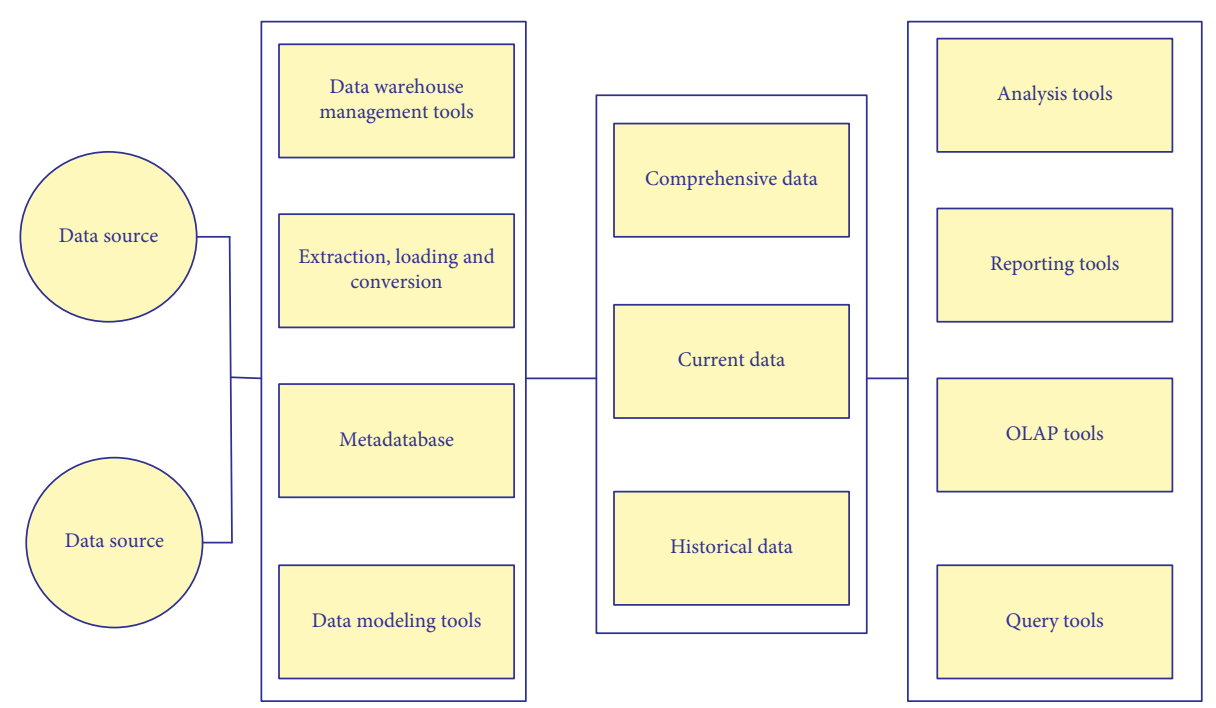

FIGURE 2: Database collection, query, and analysis system diagram.

TABLE 1: The proportion of people's sports types.

\begin{tabular}{|c|c|c|}
\hline Types of motion & Specific sports & Proportion of people (\%) \\
\hline \multirow{6}{*}{ Aerobic exercise } & Yoga & 35 \\
\hline & Swimming & 25 \\
\hline & Ride a bike & 41 \\
\hline & Jogging & 47 \\
\hline & Tai chi & 33 \\
\hline & Square dancing & 38 \\
\hline \multirow{6}{*}{ Anaerobic exercise } & Sprint & 10 \\
\hline & Push-up & 12 \\
\hline & Long jump & 5 \\
\hline & High jump & 4 \\
\hline & Tug of war & 6 \\
\hline & Diving & 8 \\
\hline
\end{tabular}

It can be seen from Figure 3 that the proportion of people who choose aerobic exercise is not much different, but it far exceeds the proportion of people who choose anaerobic exercise. Therefore, people still prefer to choose aerobic exercise. During this exercise, oxygen can completely burn sugar in the body (i.e., oxidize), consume body fat, strengthen and improve cardiopulmonary function, prevent osteoporosis, and adjust mental state. This is the main exercise method.

This article divides sports venues into gymnasiums, school playgrounds, and parks. The exercise duration is divided into three time periods: less than 30 minutes, 30-60 minutes, and more than 60 minutes. From the data in Table 2, it can be seen that the proportion of people exercising in the school playground is the highest, reaching $38 \%$, the proportion of people in the gym is $34 \%$, and the proportion of people in the park is $28 \%$. Most of the people who exercise in the school playground are college students and middleaged and elderly people living near colleges and universities. Their awareness of exercise is relatively high, and the school playground is a very convenient place for sports. The gym is mainly a place for young people to exercise, but the funds of young people are limited, so the proportion is not particularly high; the park is mainly a place for the elderly to keep fit. One is the company of three to five groups of elderly people, and the other is It is quiet and airy, so it is loved by the elderly.

It can be seen from Figure 4 that $15 \%$ of the exercise time in the gym is less than 30 minutes, $35 \%$ of the time is $30-60$ minutes, and $48 \%$ of the time is more than 60 minutes; an exercise in the school playground: the proportion of time less than 30 minutes is $25 \%$, the proportion of time between 3060 minutes is $46 \%$, and the proportion of time exceeding 60 minutes is $12 \%$; the proportion of fitness in the park is less than 30 minutes is $26 \%$. The ratio of $30-60$ minutes is $33 \%$, and the ratio of more than 60 minutes is $13 \%$.

4.2. Constructing Sports Multi-Index Data Based on 5G IoT. It can be seen from Table 3 and Figure 5 that the body fat rate of general physical education students is different from that of general people. The overall height, weight, and body fat percentage have specific characteristics, but there is almost no difference between the two. 


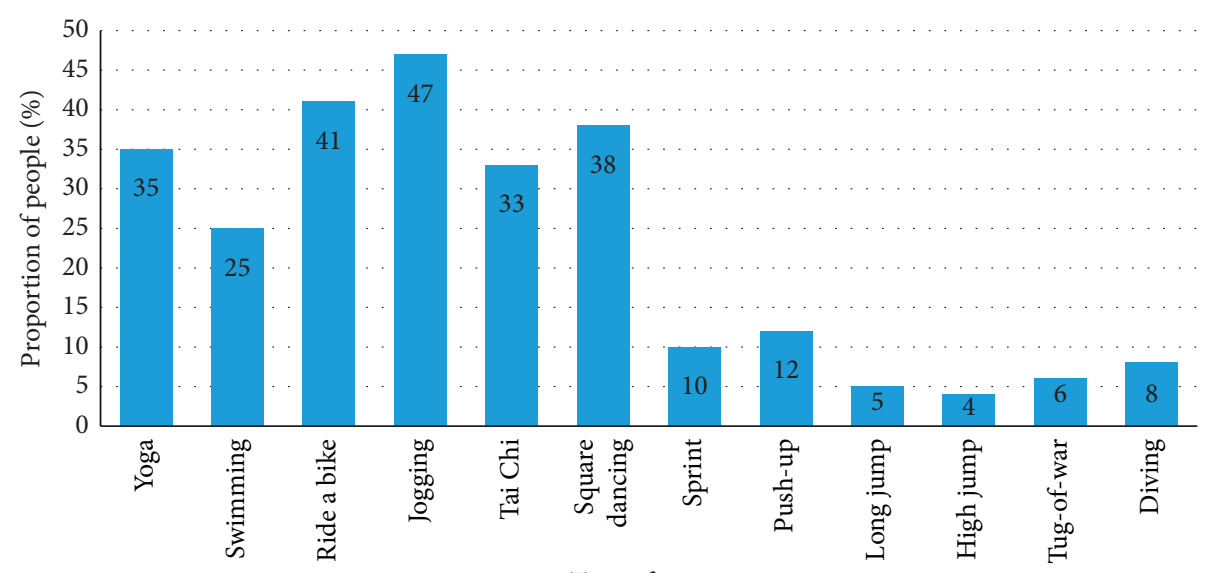

Type of sports

Proportion of people (\%)

FIgURE 3: The proportion of people's sports types.

TABLE 2: Selection of sports duration and sports venues.

\begin{tabular}{|c|c|c|c|}
\hline Sports venues & Proportion of people (\%) & Exercise duration (min) & Proportion of people (\%) \\
\hline \multirow{3}{*}{ Gym } & \multirow{3}{*}{34} & Less than 30 & 15 \\
\hline & & $30-60$ & 35 \\
\hline & & Above 60 & 48 \\
\hline \multirow{3}{*}{ School playground } & \multirow{3}{*}{38} & Less than 30 & 25 \\
\hline & & $30-60$ & 46 \\
\hline & & Above 60 & 12 \\
\hline \multirow{3}{*}{ Park } & \multirow{3}{*}{28} & Less than 30 & 26 \\
\hline & & $30-60$ & 33 \\
\hline & & Above 60 & 13 \\
\hline
\end{tabular}

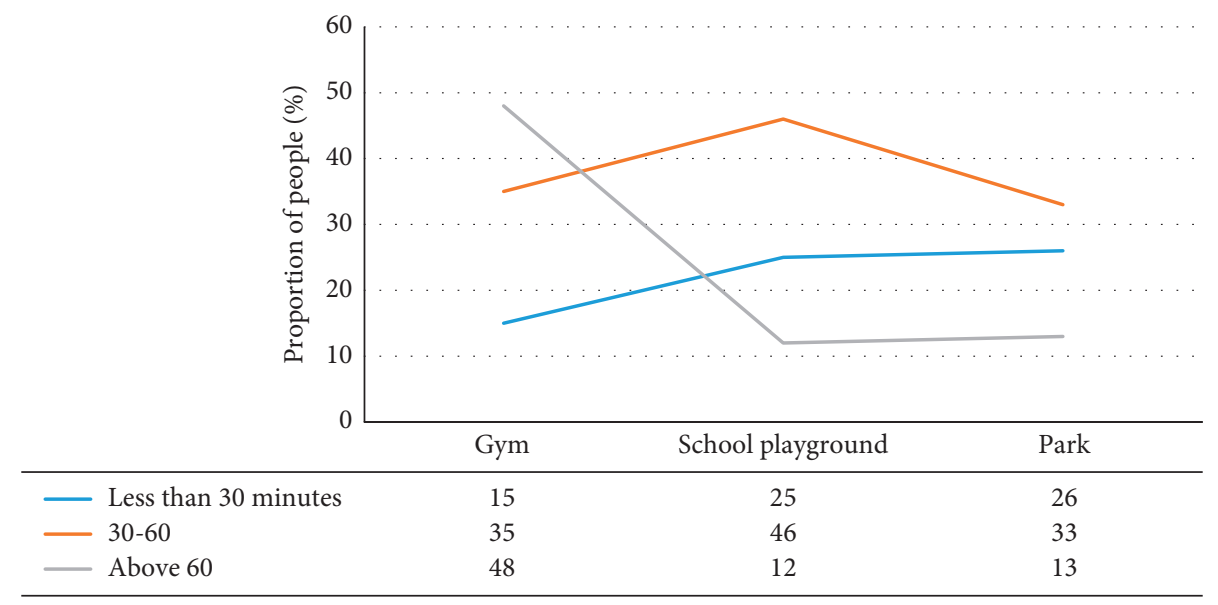

Figure 4: Proportion of people's exercise time in different sports venues.

It can be seen from Table 4 and Figure 6 that the load index of exercise volume is $38.1 \%$, the average heart rate is 122.5 pulses/minute, and the practice density in the classroom is $31.6 \%$. The appropriate pulse rate for physical therapy is 125-155 pulses/minute, and the appropriate normal load index is $40 \%-60 \%$. The indicator light is slightly lower than the corresponding pulse and load indicator, indicating that the normal load intensity is relatively low.

Table 5 and Figure 7 show that the average heart rate in exercise classes is 136.3 beats per minute. This is higher than the average heart rate of 123.3 beats per minute. The average normal load index is $55.4 \%$, which is higher than the total 
TABLE 3: Statistics of body shape index of sports students in a university.

\begin{tabular}{lccc}
\hline & Height $(\mathrm{cm})$ & Weight $(\mathrm{kg})$ & Body fat rate $(\%)$ \\
\hline \multirow{2}{*}{ Boy } & $170.1 \pm 4.48$ & $61.2 \pm 6.18$ & $12.0 \pm 3.66$ \\
& $175.2 \pm 5.81$ & $61.8 \pm 7.13$ & $12.9 \pm 3.78$ \\
\hline \multirow{2}{*}{ Girl } & $159.2 \pm 3.46$ & $49.1 \pm 4.58$ & $11.23 \pm 3.45$ \\
& $165.3 \pm 4.58$ & $52.2 \pm 5.71$ & $11.58 \pm 2.79$ \\
\hline
\end{tabular}

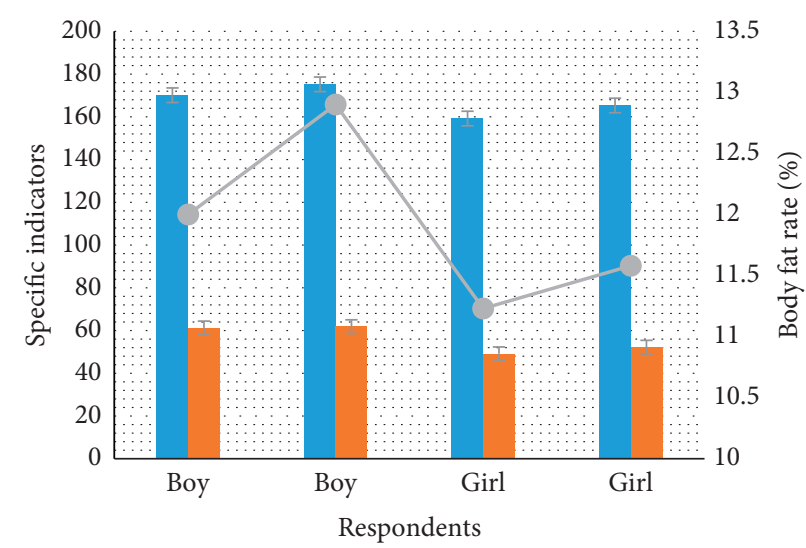

FIGURE 5: Statistics of body shape index of sports students in a university.

TABle 4: Average value of physiological burden and density of students in physical education demonstration class in a school.

\begin{tabular}{cccccccc}
\hline Gender & $\begin{array}{c}\text { Project } \\
\text { classification }\end{array}$ & $\begin{array}{c}\text { Average pulse rate of } \\
\text { class hour (times/min) }\end{array}$ & $\begin{array}{c}\text { Preclass intravenous } \\
\text { infusion rate (times/min) }\end{array}$ & $\begin{array}{c}\text { Maximum heart } \\
\text { rate (times/min) }\end{array}$ & $\begin{array}{c}\text { Physiological } \\
\text { burden index } \\
\text { (\%) }\end{array}$ & $\begin{array}{c}\text { Physiological } \\
\text { burden level }\end{array}$ \\
\hline \multirow{6}{*}{ Boy } & Track and field & 143.5 & 89.2 & 183.9 & 59.4 & Middle & 26.2 \\
& Gymnastics & 122.6 & 87.1 & 194 & 38.2 & Small & 31.7 \\
& Ball game & 123.6 & 77.8 & 183 & 44.9 & Middle & 46.9 \\
& Taekwondo & 108.1 & 67.1 & 163 & 36.4 & Small & 56.1 \\
\hline \multirow{2}{*}{ Girl } & Track and field & 128.1 & 85.2 & 167 & 45.4 & Middle & 63 \\
& Taekwondo & 135.1 & 90.1 & 181 & 51.0 & Middle & - \\
\hline
\end{tabular}

load index of $42.4 \%$. This shows that the load intensity of the segment movement process is significantly higher than the whole process. In addition, from the overall index data of the complete course, the average heart rate of men in taekwondo, basketball, and gymnastics is less than 125 pulses, and the usual load index is only $30.2 \%$ of the actual density of the demonstration class, which affects the intensity of practice.

In Table 6, we divide the physical education curriculum into large, medium, and small load courses and use the currently commonly used evaluation standards, namely the comprehensive index method, the average heart rate method, and the heart rate index method, to comprehensively evaluate the sensitivity, specificity, and Youden index of exercise load. Sensitivity refers to the proportion of suitable people who can be identified as patients by the screening method. Specificity refers to the proportion of people who can be correctly defined as nonpatients through screening methods. Youden index is a method for evaluating the reliability of screening tests. When false positives are considered equally harmful, you can apply the Yoden Index. From Table 6, we can see that the comprehensive coincidence rates of these three methods are $58.43 \%, 47.99 \%$, and $57.47 \%$. Among them, the average heart rate and comprehensive index method and the expert system coincide with rates close to $60 \%$, which shows that the large, medium, and small load arrangements arranged by the two methods are appropriate.

It can be seen from the data in Table 7 that the most injured part is the finger, accounting for $17.13 \%$, which fully reflects the fragility of the human finger. If it receives a horizontal or vertical impact, it is very easy to cause damage to the finger. Then the ankles, thighs, and buttocks were the most injured, the proportions reached $14.76 \%, 13.63 \%$, $11.57 \%$, and the proportion of injuries all exceeded $10 \%$. As can be seen from Figure 8, the ankle is very easy to cause crippled feet during exercise, so be sure to warm up before exercise. The thighs and buttocks are parts that people are not easy to notice during daily exercise, so the injury rate is relatively high. 


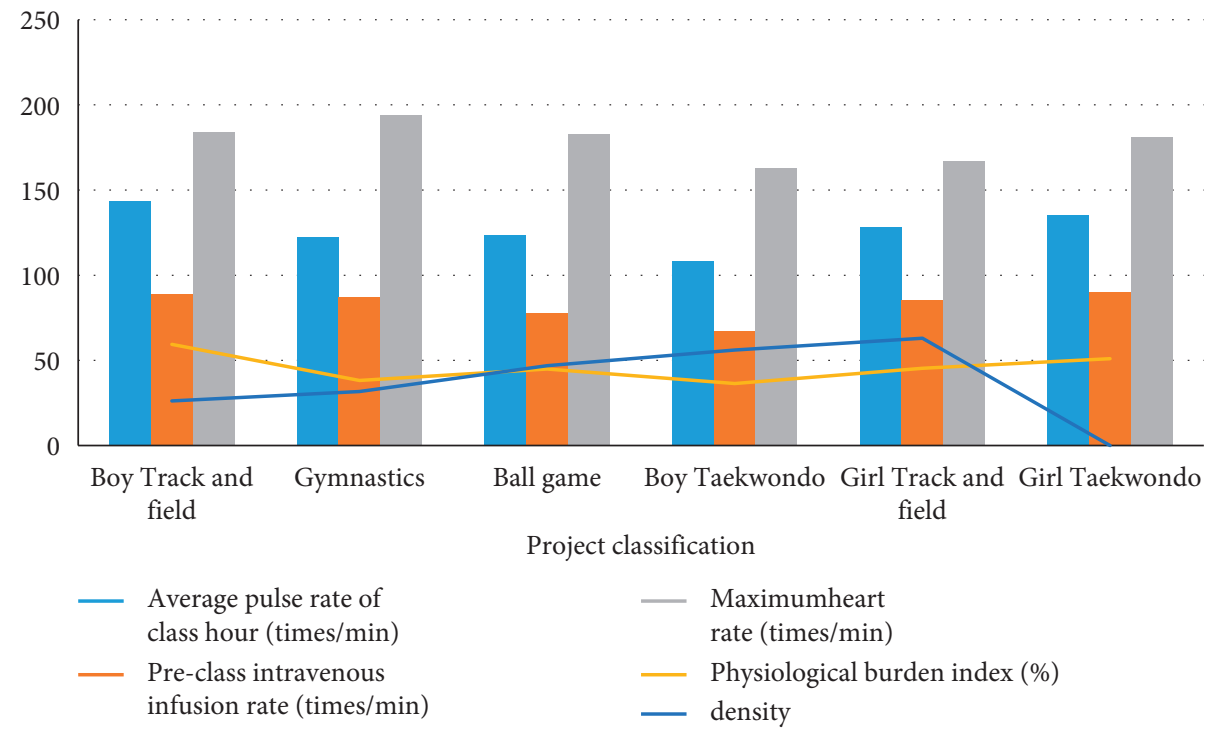

FIgURE 6: Average value of physiological burden and density of students in physical education demonstration class in a school.

TABLE 5: Complete display of physiological load and density test results after class.

\begin{tabular}{|c|c|c|c|c|c|c|c|}
\hline Gender & Content & $\begin{array}{l}\text { Average pulse rate of } \\
\text { class hour } \\
\text { (times/min) }\end{array}$ & $\begin{array}{l}\text { Preclass intravenous } \\
\text { infusion rate } \\
\text { (times/min) }\end{array}$ & $\begin{array}{l}\text { Maximum heart } \\
\text { rate (times } / \mathrm{min} \text { ) }\end{array}$ & $\begin{array}{l}\text { Physiological } \\
\text { burden index } \\
(\%)\end{array}$ & $\begin{array}{l}\text { Physiological } \\
\text { burden level }\end{array}$ & Density \\
\hline \multirow{5}{*}{ Boy } & $\begin{array}{c}\text { Track and field } \\
\text { mean value }\end{array}$ & 139.6 & 95.6 & 187.0 & 52.1 & Middle & 30.3 \\
\hline & $\begin{array}{c}\text { Gymnastics mean } \\
\text { value }\end{array}$ & 122.6 & 87.1 & 193.0 & 38.2 & Small & 31.7 \\
\hline & $\begin{array}{l}\text { Ball game mean } \\
\text { value }\end{array}$ & 117.1 & 78.4 & 172.9 & 40.9 & Middle & 56.5 \\
\hline & $\begin{array}{c}\text { Taekwondo mean } \\
\text { value }\end{array}$ & 109.0 & 67.1 & 162.1 & 42.7 & Middle & 56.1 \\
\hline & Total average & 123.4 & 83.9 & 180.3 & 42.5 & Middle & 43.7 \\
\hline
\end{tabular}

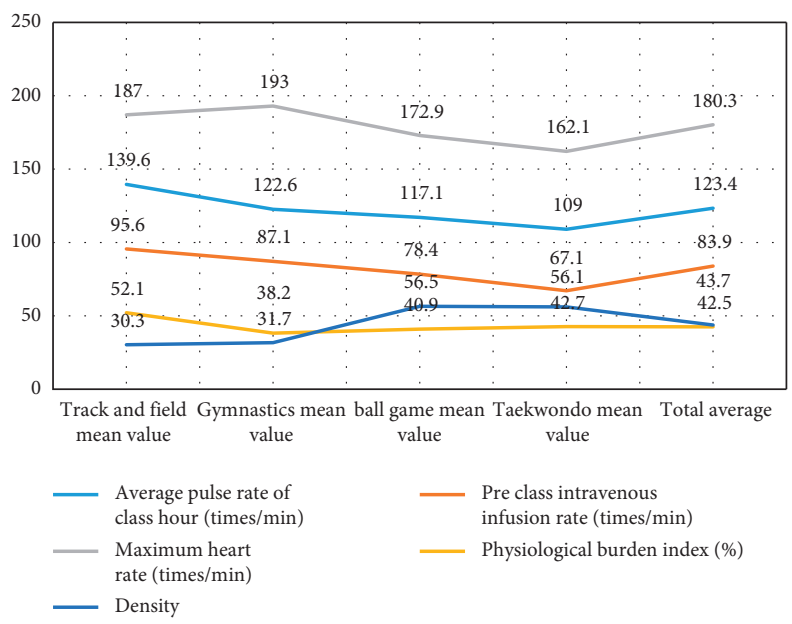

Figure 7: Complete display of physiological load and density test results after class. 
TABLE 6: Comparison of sensitivity, specificity, and Youden index of different methods in evaluating exercise load.

\begin{tabular}{lcccccccccc}
\hline $\begin{array}{l}\text { Evaluation } \\
\text { method }\end{array}$ & \multicolumn{3}{c}{ Heavy load } & \multicolumn{4}{c}{ Medium load } & \multicolumn{3}{c}{ Small load } \\
Sensitivity & Specificity & Youden & Sensitivity & Specificity & Youden & Sensitivity & Specificity & Youden & compliance rate \\
\hline $\begin{array}{l}\text { Average heart rate } \\
\text { method }\end{array}$ & 38.8 & 88.4 & 28.1 & 75.1 & 47.6 & 22.7 & 54.8 & 98.5 & 53.2 & 58.43 \\
\hline $\begin{array}{l}\text { Heart rate index } \\
\text { method }\end{array}$ & 80.7 & 58.0 & 37.8 & 23.5 & 72.4 & -5.3 & 40.7 & 93.8 & 34.5 & 47.99 \\
$\begin{array}{l}\text { Composite index } \\
\text { method }\end{array}$ & 63.9 & 82.1 & 44.9 & 56.4 & 58.8 & 15.1 & 53.2 & 95.3 & 48.4 & 57.47 \\
\hline
\end{tabular}

TABLe 7: Distribution of injured parts in sports.

\begin{tabular}{lcc}
\hline Injured site & Number of people & Proportion (\%) \\
\hline Finger & 46 & 17.13 \\
Ankle & 42 & 14.76 \\
Lap & 39 & 13.63 \\
Thigh & 33 & 11.57 \\
Waist & 28 & 9.77 \\
Wrist & 24 & 8.34 \\
Upper arm & 18 & 6.19 \\
Elbow & 13 & 4.31 \\
Forearm & 11 & 3.68 \\
Calf & 10 & 3.36 \\
Hip & 9 & 2.88 \\
Back & 7 & 2.25 \\
Buttocks & 5 & 1.44 \\
Other & 8 & 2.52 \\
\hline
\end{tabular}

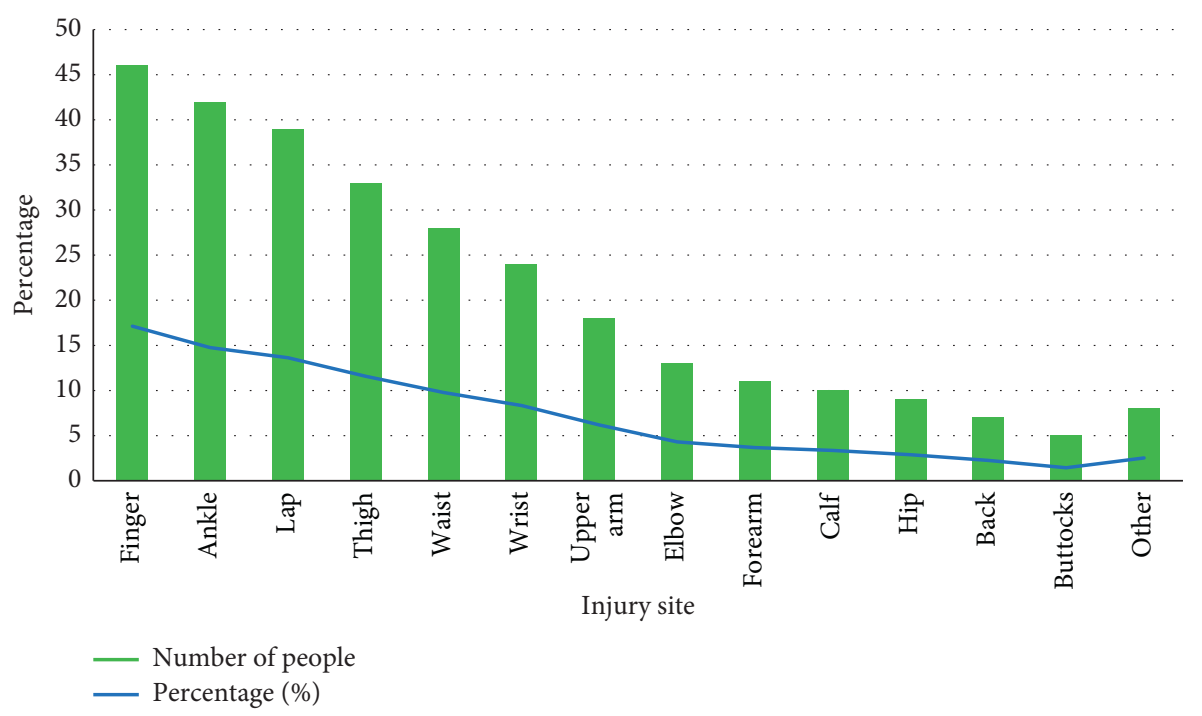

FIGURE 8: Distribution of injured parts in sports.

\section{Conclusion}

With the advent of the $5 \mathrm{G}$ era, more and more public welfare cases of the successful integration of $5 \mathrm{G}$ and sports have emerged, and the integration of $5 \mathrm{G}$ technology applications and the sports industry has become a general trend. The advent of $5 \mathrm{G}$ has given birth to new smart sports models such as sports content technology, 5G-covered venue features, and 5G high-definition live broadcast. With the advancement of new technologies such as $5 \mathrm{G}$, the sports industry, with the blessing of $5 \mathrm{G}$, has gradually broken through the traditional model, found a new revolution, and achieved a new pace in sports development. This article mainly studies the analysis and discussion of the construction of sports multi-index data based on 5G IoT. By consulting related documents, we can deeply understand and master the key technologies of the IoT and apply them to the analysis of sports multi-index data. This article mainly 
adopts the data mining and analysis technology in the IoT, selects the data needed in this article from the distributed memory massive database, and combines the deep learning algorithm and artificial neural network algorithm to analyze people's sports item preferences, exercise duration, various physical indicators during exercise, and the distribution of sports injuries.

The innovations of this article are that, first, qualitative analysis and quantitative analysis are combined to fully analyze research data; second, theoretical research and empirical research are combined, based on the Internet of Things technology model, combined with sports, carry out case analysis on the actual situation in the article.

There are still some shortcomings in this article. The main reason is that the IoT is less used in sports, and there may be running-in problems in actual operation. This article analyzes sports with multiple indicators, and the selection of indicators is not comprehensive enough. It needs to be more perfected. Nowadays, more and more people are concerned about sports and the health of themselves and their families. Sports career has received more support. It is believed that sports based on 5G IoT technology will develop better and better.

\section{Data Availability}

The data that support the findings of this study are available from the corresponding author upon reasonable request.

\section{Conflicts of Interest}

The authors declare that they have no conflicts of interest.

\section{Acknowledgments}

This work was supported by Xinjiang Sports Culture Research Center (XJNURWJD2019B09) and Xinjiang Normal University Public Health and Health Research Program (XJNUZX202005).

\section{References}

[1] M. S. Balaji and S. K. Roy, "Value co-creation with Internet of things technology in the retail industry," Journal of Marketing Management, vol. 33, no. 1-2, pp. 7-31, 2017.

[2] J.-H. Park and B.-K. Chun, "The influence of social support on self-determination and athletic performances of winter sports athlete," Korean Journal of Sports Science, vol. 26, no. 4, pp. 317-330, 2017.

[3] M. R. Palattella, M. Dohler, A. Grieco et al., "Internet of things in the $5 \mathrm{G}$ era: enablers, architecture, and business models," IEEE Journal on Selected Areas in Communications, vol. 34, no. 3, pp. 510-527, 2016.

[4] T. K. L. Hui, R. S. Sherratt, and D. D. Sanchez, "Major requirements for building smart homes in smart cities based on Internet of things technologies," Future Generation Computer Systems, vol. 76, pp. 358-369, 2016.

[5] Z. H. Wu, "Research on the application of Internet of things technology to digital museum construction," Acta Geoentica Sinica, vol. 38, no. 2, pp. 293-298, 2017.
[6] N. Hrynchak, "Analysis of the impact of the Internet of things technology on the development of the transport and logistics market," Scientific Bulletin of the National Academy of Statistics, Accounting and Audit, vol. 4, pp. 74-82, 2020.

[7] S. R. Chandra and W. Yafeng, "Cloud Things Constructionthe integration of Internet of things and cloud computing," Future Generation Computer Systems, vol. 56, pp. 684-700, 2016.

[8] C. Aranzazu-Suescun and M. Cardei, "Distributed algorithms for event reporting in mobile-sink WSNs for Internet of things," Tsinghua Science and Technology, vol. 22, no. 4, pp. 413-426, 2017.

[9] A. Caputo, G. Marzi, and M. M. Pellegrini, “The Internet of things in manufacturing innovation processes: development and application of a conceptual framework," Business Process Management Journal, vol. 22, no. 2, pp. 383-402, 2016.

[10] C. Chang, S. N. Srirama, and R. Buyya, "Mobile cloud business process management system for the Internet of things: a survey," ACM Computing Surveys, vol. 49, no. 4, pp. 1-42, 2016.

[11] G. N. L. Diega, "Clouds of Things. Data protection and consumer law at the intersection of cloud computing and the Internet of Things in the United Kingdom," Journal of Law \& Economic Ragulation, vol. 9, no. 1, pp. 69-93, 2016.

[12] S. Wan, Y. Zhao, T. Wang et al., "Multi-dimensional data indexing and range query processing via Voronoi diagram for Internet of things," Future Generation Computer Systems, vol. 91, pp. 382-391, 2018.

[13] M. B. Mollah, M. A. K. Azad, and A. Vasilakos, "Secure data sharing and searching at the edge of cloud-assisted Internet of things," IEEE Cloud Computing, vol. 4, no. 1, pp. 34-42, 2017.

[14] S. Feng, P. Setoodeh, and S. Haykin, "Smart home: cognitive interactive people-centric Internet of things," IEEE Communications Magazine, vol. 55, no. 2, pp. 34-39, 2017.

[15] K. Wang, X. Qi, L. Shu, D.-j. Deng, and J. J. P. C. Rodrigues, "Toward trustworthy crowdsourcing in the social Internet of things," IEEE Wireless Communications, vol. 23, no. 5, pp. 30-36, 2016.

[16] E. Sisinni, A. Saifullah, S. Han, U. Jennehag, and M. Gidlund, "Industrial internet of things: challenges, opportunities, and directions," IEEE Transactions on Industrial Informatics, vol. 14, no. 11, pp. 4724-4734, 2018.

[17] R. Rajiv, R. Omer, N. Surya et al., "The next grand challenges: integrating the Internet of things and data science," IEEE Cloud Computing, vol. 5, no. 3, pp. 12-26, 2018.

[18] Z. Lv, A. K. Singh, and J. Li, "Deep learning for security problems in $5 \mathrm{G}$ heterogeneous networks," IEEE Network, vol. 35, no. 2, pp. 1-8, 2021.

[19] Q. Xue, Y. Zhu, and J. Wang, "Joint distribution estimation and naïve bayes classification under local differential privacy," IEEE Transactions on Emerging Topics in Computing, vol. 99, p. $1,2020$.

[20] X. Li, Y. Zhu, and J. Wang, "Highly efficient privacy preserving location-based services with enhanced one-round blind filter," IEEE Transactions on Emerging Topics in Computing, vol. 99, pp. 1-12, 2019.

[21] I. Azimi, A. M. Rahmani, P. Liljeberg, and H. Tenhunen, "Internet of things for remote elderly monitoring: a study from user-centered perspective," Journal of ambient intelligence and humanized computing, vol. 8, no. 2, pp. 273-289, 2017.

[22] A. V. Kolot, "Modern problems of perfection of elite light athletic sportsmen's technical skillfulness perfection," 
Pedagogics psychology medical-biological problems of physical training and sports, vol. 20, no. 2, pp. 26-33, 2016.

[23] M. Dehghani, A. Delbar Saf, A. Vosoughi, G. Tebbenouri, and H. Ghazanfari Zarnagh, "Effectiveness of the mindfulnessacceptance-commitment-based approach on athletic performance and sports competition anxiety: a randomized clinical trial," Electronic Physician, vol. 10, no. 5, pp. 6749-6755, 2018.

[24] M. P. Zoland, M. E. Maeder, J. C. Iraci, and D. A Klein, "Referral patterns for chronic groin pain and athletic pubalgia/sports hernia: magnetic resonance imaging findings, treatment, and outcomes," American Journal of Orthopedics, vol. 46, no. 4, pp. E251-E256, 2017.

[25] A. C. Thomas and J. B. Driban, "Athletic trainers' osteoarthritis consortium: raising awareness of osteoarthritis in the sports medicine community," International Journal of Athletic Therapy \& Training, vol. 22, no. 5, pp. 1-3, 2017.

[26] Z. Rahmat and I. Irfandi, "Evaluation of management training athletic sports club of run, jump and floor in sport and youth office (diaspora) aceh," Budapest International Research and Critics in Linguistics and Education (BirLE) Journal, vol. 2, no. 2, pp. 321-329, 2019.

[27] T. Alexandra, "Contemporary administration of Greek Olympic and athletic sports organizations," International Journal of Scientific Research, vol. 6, no. 8, pp. 315-317, 2017.

[28] C. Helma, T. Cramer, S. Kramer et al., "Data mining and machine learning techniques for the identification of mutagenicity inducing substructures and structure activity relationships of noncongeneric compounds," Journal of Chemical Information and Computer Sciences, vol. 35, no. 4, pp. 1402-1411, 2018. 